\title{
A NEW ALGORITHM CALCULATING THE DISTANCE BETWEEN BINARY ARBORESCENCES
}

1. Procedure declaration. An arborescence $A$ is defined as a directed tree that has a root, i.e., a tree with the vertex $v_{0}$, such that all vertices of the tree can be reached by a path starting from $v_{0}$. Let $X=\{1,2, \ldots, n\}$ be the set of pendant (terminal) vertices of an arborescence $A$, i.e., $d^{-}(i)=1$ and $d^{+}(i)=0$ for $i=1,2, \ldots, n$, where $d^{-}(i)$ and $d^{+}(i)$ denote the inner demi-degree and the outer demi-degree of the vertex $i$, respectively. By a binary arborescence we mean such an arborescence in which $d^{+}(v)=2$ for each $v \notin X$. Since in this paper we consider only binary arborescences, the word "binary" is omitted.

The procedure arbmetric calculates the distance between arborescences (more precisely, the distance between hypergraphs generated by arborescences) with the same set of pendant vertices, proposed in [1], and is a modification of the procedure hypergraphmetrics presented in [2].

The arborescence $A$ generates the hypergraph $H_{A}=\left(X, \mathscr{E}_{A}\right)$, where the class of edges $\mathscr{E}_{A}$ is defined as follows: each non-pendant vertex $v$ generates exactly one edge in $\mathscr{E}_{A}$ which consists of those elements of the set $X$ which are the pendant vertices of the subarborescence generated by the vertex $v$. The distance between $H_{A_{1}}=\left(X, \mathscr{E}_{A_{1}}\right)$ and $H_{A_{2}}=\left(X, \mathscr{E}_{A_{2}}\right)$ was defined in [1] as

$$
\varrho\left(H_{A_{1}}, H_{A_{2}}\right)=\frac{1}{n-1} \min _{p \in \mathscr{F}} \sum_{i=1}^{n-1} \frac{\left|E_{i} \Delta F_{p(i)}\right|}{\left|E_{i} \cup F_{p(i)}\right|},
$$

Where $p(i)$ is the $i$-th element of the permutation $p$ of the first $n-1$ integers, $\mathscr{P}$ is the set of all such permutations, and $E_{i} \in \mathscr{E}_{A_{1}}, F_{p(i)} \in \mathscr{E}_{A_{2}}$, $i=1,2, \ldots, n-1$.

Now, let us describe a new element of the algorithm given in this paper, which is a better representation of a hypergraph by some vector. First, consider the example of the arborescence presented in Fig. 1. 
This arborescence can be represented by the hypergraph $H_{A}=\left(X, \mathscr{E}_{A}\right)$ where $X=\{1,2, \ldots, 9\}$ and the class of edges $\mathscr{E}_{A}$ may be the following:

$$
\begin{gathered}
E_{1}=\{4,5\}, \quad E_{2}=\{2,7\}, \quad E_{3}=\{8,9\}, \quad E_{4}=E_{1} \cup\{1\}, \\
E_{5}=E_{2} \cup\{6\}, \quad E_{6}=E_{5} \cup\{3\}, \quad E_{7}=E_{3} \cup E_{6}, \quad E_{8}=E_{4} \cup E_{7} .
\end{gathered}
$$

Now, note that we are always able to form the class $\mathscr{E}_{A}$ in such a way that

(a) $\left|E_{1}\right|=2,\left|E_{i}\right| \leqslant\left|E_{i+1}\right|(i=1,2, \ldots, n-2)$,

(b) each successive edge can be either

$1^{\circ}$ the set of two different vertices, or

$2^{\circ}$ the sum of the edge already defined and of a new vertex, or

$3^{0}$ the sum of two edges already defined.

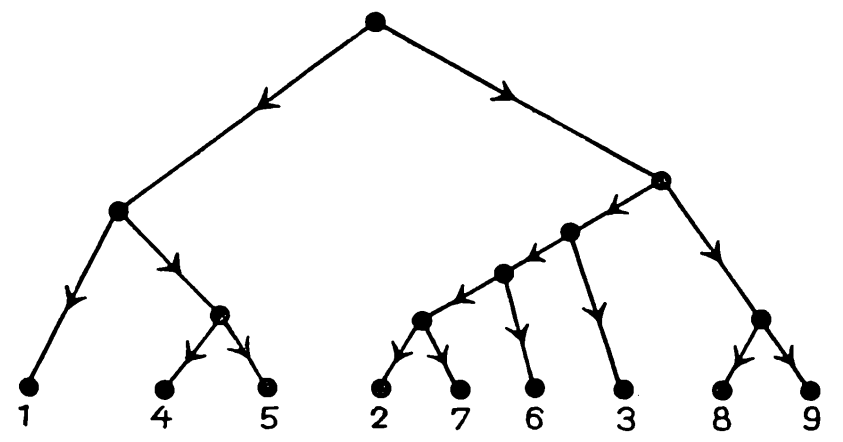

Fig. 1

For a hypergraph generated by a binary arborescence, no other situation is possible. Thus, we can represent each of the $n-1$ edges of $H_{A}$ by two numbers only, which are either

$1^{\circ}$ numbers of vertices, or

$2^{\circ}$ the index (with a minus sign) of the edge already defined and the number of a new vertex, or

$3^{\circ}$ two indices (with a minus sign) of the edges already defined.

The components of the vector representing the arborescence given in Fig. 1 are the following (for clarity we write each edge in parentheses):

$(4, \tilde{\jmath}),(2,7),(8,9),(-1,1),(-2,6),(-5,3),(-3,-6),(-4,-7)$.

Therefore, we need only $2 n-2$ elements as components of the vector representing an arborescence, whereas the number of components needed in the previous algorithm [2] was much greater for large arborescences.

Data: $A 1[1: 2 m]$, $m$ - the number of pendant vertices minus 1 , i.e., $m=n-1$; $A 2[1: 2 m]$ - arrays representing the first and the second arborescences, respectively; 
precedure arbmetric (m, $A 1, A 2, d, f a c$, inf, as8ign);

value $m$, fac, inf;

integer $\mathrm{m}$, fac, inf;

resid d

integer ray A1, A2;

procedure asøign;

begin

integer b1, b2, $1, j, k, k 1, k 2,1,11,12,8, w$;

integer array $B 1, B 2[1: m]$;

precedure size $(m, I, A, B)$;

rolue $\mathrm{m}$;

integer $\mathrm{m}, \mathrm{I}$;

integer array $A, B$;

begin

Integer $a, b, i, j, k$;

$B[1]:=1:=2$;

j: $:=3$;

for 1:=2 ster 1 until m do

begin

$$
\begin{aligned}
& a:=A[j] ; \\
& b:=A[j+1] ; \\
& \text { if } a>0 \\
& \text { then } \\
& \text { begin } \\
& B[i]:=2 ; \\
& 1:=1+2 ; \\
& \text { ge to ET } \\
& \text { end } a>0
\end{aligned}
$$

else

If $b>0$ 


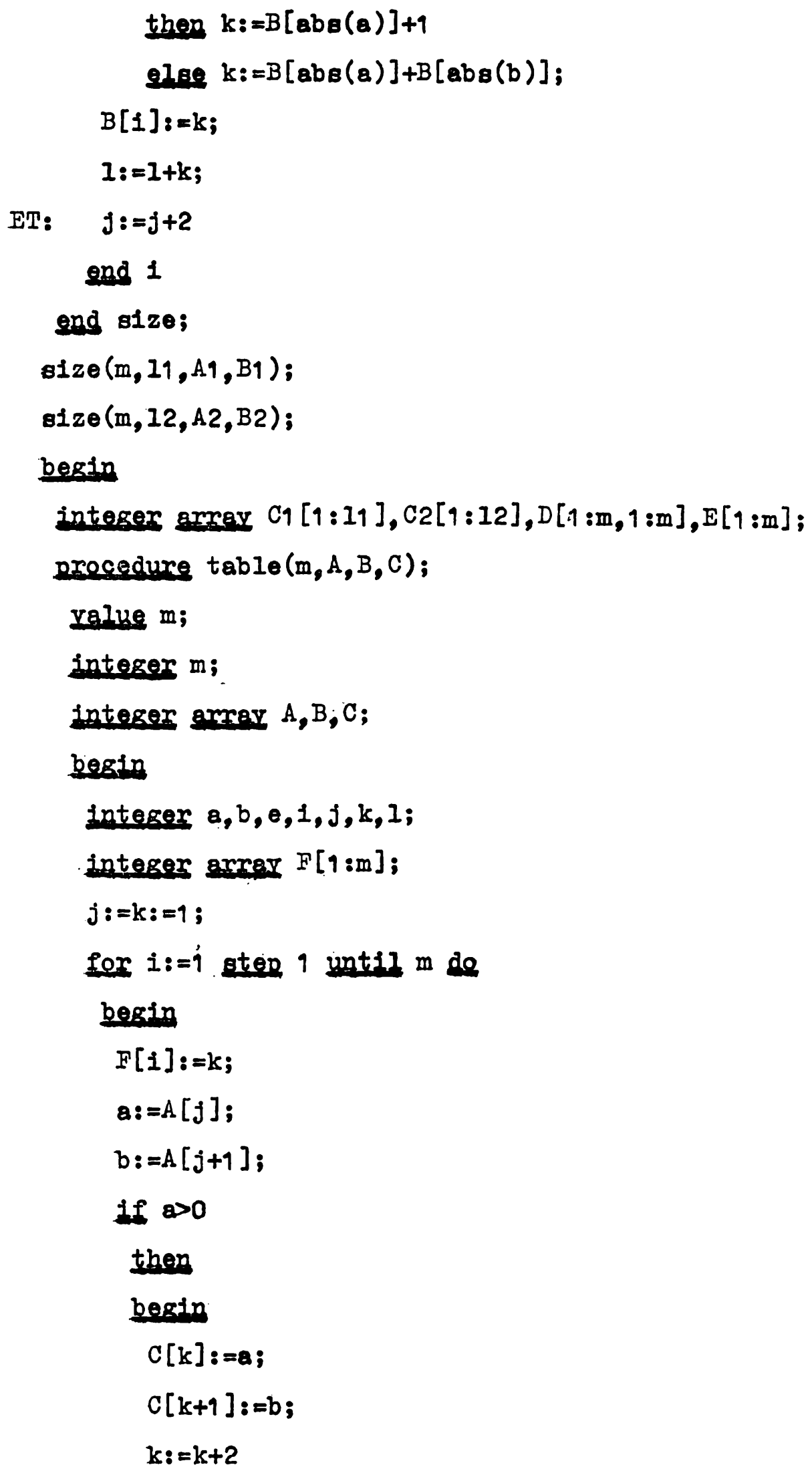


end $a>0$

\section{else}

begtin

B: $=a_{B}(a)$;

$\theta:=F[a]$;

$8:=k+B[a]-1 ;$

for $1:=k$ sten 1 until a do

begin

$c[1]:=C[\theta]$;

$e:=e+1$

end 1;

iff $b>0$

then

begth

$c[a+q]:=b_{1}$

$k:=8+2$.

end $b>0$

\section{else}

begin

$\mathrm{b}:=\mathrm{abs}(\mathrm{b})$;

$\theta:=F[b]$;

$\mathrm{b}:=a+B[b] ;$

for $1:=a+1$ steg 1 until $b$ do

begin

$C[1]:=C[e]$;

$e:=e+1$

end 1;

$k:=b+1$

end $b<0$

end $a<0$; 


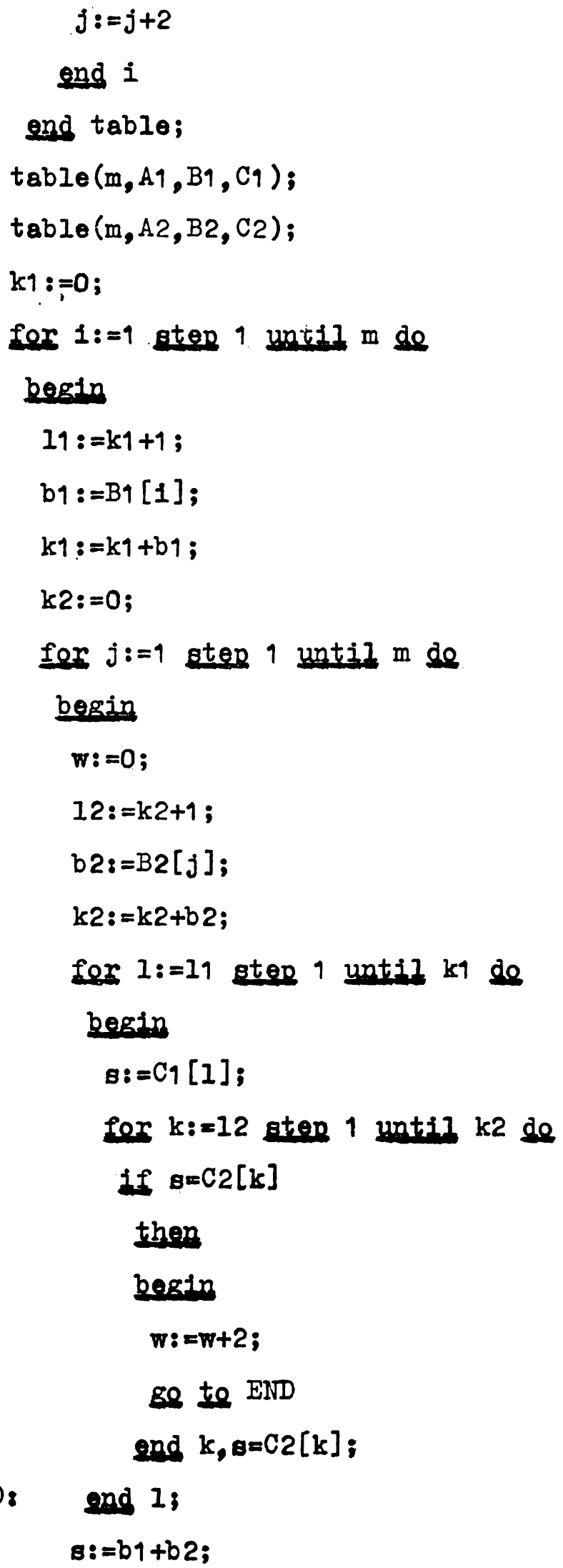




\title{
$D[i, j]:=(8-w) /(8-w+2) \times f a c$
}

\author{
end $j$ \\ end 1; \\ assign (m, $D, D, 8$, Inf $)$; \\ $\mathrm{d}:=\boldsymbol{g} /(\mathrm{m} \times \mathrm{fac})$
}

end

\section{end arbmetric}

fac - the factor by which the cost matrix $c$ (see the procedure assign) must be multiplied in order to obtain the integer coefficients of $c$;

inf - the maximal allowed number of type integer in the computer;

assign - the procedure with the following procedure head:

procedure assign ( $m, c, p$, total, inf);

value $m$, inf;

integer $m$, total, inf;

integer array $c, p$;

Result:

this procedure is published in [3] and finds $\min \sum_{i=1}^{m} c_{i p_{i}}$, where $\left(p_{1}, p_{2}, \ldots, p_{m}\right)$ is the permutation of the numbers $(1,2, \ldots, m)$.

- the distance between arborescences.

2. Method used. The method is similar to that in [2].

3. Certification. The procedure has been verified on the Odra 1204 computer for the problems described in [1].

\section{References}

[1] M. Karoński and Z. Palka, On Marczewski-Steinhaus type distance between hypergraphs, Zastos. Mat. 16 (1977), p. 47-57.

[2] - Algorithm 69: Two distances between hypergraphs, ibidem 16 (1980), p. 671-679.

[3] J. Kucharczyk and M. Sysło, Algorytmy optymalizacji w jezyku ALGOL 60. Warszawa 1975.

MATHEMaticaL INSTITUTE

A. MICKIEWICZ UNIVERSITY

$60 \cdot 769$ POZNAN 